\title{
Relevancia del puntaje de Rotterdam para el pronóstico de empeoramiento en pacientes con traumatismo encéfalocraneano
}

Ariel Varela Hernández¹, Claudio Martínez Terreu ${ }^{1}$, Rodolfo Muñoz Gajardo¹, Reinaldo Torres Aravena ${ }^{1}$, Félix Orellana Cortez ${ }^{1}$,Patricio Herrera Astudiño ${ }^{1}$,Luis Lamus Aponte ${ }^{1}$

1 Neurocirujanos, Servicio de Neurocirugía, Hospital Regional de Talca. Chile.

Rev. Chil. Neurocirugía 44: 114-120, 2018

\section{Resumen}

Introducción: El puntaje tomográfico de Rotterdam ha mostrado mejores rendimientos en cuanto al pronóstico de los resultados de la atención de los pacientes con traumatismo encéfalocraneano al compararla con la de Marshall, sin embargo, no ha sido aún evaluada para la predicción del empeoramiento de las lesiones intracraneales detectadas en la tomografía computarizada inicial de cráneo. Material y Método: Se realizó un estudio prospectivo con los pacientes hospitalizados por traumatismo encéfalocraneano en el Hospital Universitario "Manuel Ascunce Domenéch", Camagüey, Cuba, desde el 1 de julio de 2011 al 31 de enero de 2012. Se incluyeron los enfermos evaluados con tomografía computarizada de cráneo en el servicio de urgencia y se repitió este examen como control durante el mismo periodo de hospitalización. Se aplicaron técnicas de estadística descriptiva, regresión logística y ANOVA. Resultados: Se estudiaron 84 pacientes, y en 13 (15,5\%) se detectó empeoramiento de las lesiones en la tomografía computarizada de control que motivó la realización de tratamiento neuroquirúrgico urgente. Se demostró la relación del aumento del puntaje de Rotterdam con la ocurrencia de esta complicación. Conclusiones: Para el pronóstico de los pacientes con traumatismo encéfalocraneano no solo es relevante lo relacionado con la mortalidad o los malos resultados, sino también con el riesgo de amplificación relevante de las lesiones intracraneales detectadas en la tomografía computarizada de cráneo inicial, lo cual brinda la posibilidad de generar cambios terapéuticos más oportunos. En este sentido constituye un factor de riesgo la elevación del puntaje de Rotterdam en la TC inicial.

Palabras clave: Puntaje de Rotterdam, neuromonitoreo, traumatismo encéfalocraneano.

\section{Abstract}

Background: Rotterdam tomographic score has shown to be better than Marshall scale on the prognosis of the outcomes for patients with head trauma, but has not yet been assessed for prediction of worsening of intracranial lesions detected in the initial head computed tomography scan. Material and Method: A prospective study was conducted with patients with head trauma admitted at the Manuel Ascunce Domenéch University Hospital, Camagüey, Cuba, from July 1, 2011 to January 31, 2012. Patients evaluated with cerebral scan on admission and this test was repeated as a control during the same hospitalization period were included. Descriptive statistics techniques were applied, logistic regression and ANOVA. Results: 84 patients were studied, in $13(15.5 \%)$ initial lesions worsening on a control cerebral scan that motivated the execution of urgent neurosurgical treatment. Increase Rotterdam score relationship with the occurrence of this complication was demonstrated. Conclusions: prognosis of patients with head trauma is not only relevant for mortality or poor results, but also with the risk of significant amplification of intracranial lesions detected in the initial head tomography scan, which offers the possibility to generate more opportune therapeutic changes. In this sense, the elevation of the Rotterdam scores in the initial head tomography scan is a risk factor.

Key words: Rotterdam score, neuromonitoring, cerebral trauma. 


\section{Introducción}

En la etapa actual de atención neurointensiva de los pacientes con traumatismo encéfalocraneano (TEC), el establecimiento del pronóstico tanto a corto como a mediano plazo, constituye un elemento esencial para el cribaje inicial de los pacientes, la orientación adecuada a los familiares y el equipo sanitario, la correcta planificación de los recursos disponibles y el desarrollo de investigaciones encaminadas a mejorar los resultados terapéuticos.

De manera inicial estas herramientas han descansado sobre la información obtenida de algunas escalas clínicas como la de Glasgow, universalmente la más usada, la FOUR (Full Outline of Unresponsiveness), entre otras. Todas ellas presentan limitaciones para su aplicación en enfermos sedados, intubados o bajo el efecto de intoxicaciones exógenas; además con su empleo no es posible establecer el diagnóstico de las lesiones patoanatómicas específicas de los pacientes ${ }^{7,4}$.

Por tales motivos, el desarrollo de escalas basadas en la información estructural obtenida por algunas técnicas de neuroimágenes, sobre todo con Tomografía Computarizada (TC) de cráneo, ha mostrado una aplicación creciente en las últimas décadas y se ha llegado a determinar el valor pronóstico independiente de varias variables imagenológicas en el modelo conocido como IMPACT, acrónimo tomado del idioma inglés, que se asume como el más completo hasta el momento en relación a los pacientes con TEC. También se ha resaltado de las mismas la fácil aplicación y poca variabilidad interobservador, cuando los estudios son interpretados por personal debidamente entrenado ${ }^{1}$.

En este sentido contamos en estos momentos con la escala tomográfica de Marshall (Tabla 1), descrita en 1991, la de Rotterdam en 2005 (Tabla 2) y otras aún más recientes como la de Estocolmo y Helsinki, aún en proceso de validación externa. Empero la de Marshall se mantiene como la más usada a nivel mundial, la de Rotterdam ha mostrado mayor utilidad predictiva en cuanto a la mortalidad y los malos resultados a los 6 meses después del TEC.

Vale destacar que aunque dichas escalas se han estudiado en cuanto a la predicción de la mortalidad y los malos resultados a corto y mediano plazos,

Tabla 1.

Clasificación tomográfica de Marshall para pacientes con traumatismo encéfalocraneano

\begin{tabular}{|c|c|}
\hline Tipo & Descripción de los signos tomográficos \\
\hline 1 & Normal \\
\hline II & $\begin{array}{l}\text { Lesiones pequeñas: Cisternas presentes con luxación de línea media } \\
<5 \mathrm{~mm} \text { o lesiones de densidad presentes, no presencia de lesiones } \\
\text { hiperdensas o mixtas }>25 \mathrm{ml} \text {, puede incluir fragmentos óseos o cuerpos } \\
\text { extraños }\end{array}$ \\
\hline III & $\begin{array}{l}\text { Cisternas obliteradas: Cisternas comprimidas o ausentes, luxación de } \\
\text { línea media }<5 \mathrm{~mm} \text { o lesiones de densidad presentes, no presencia de } \\
\text { lesiones hiperdensas o mixtas }>25 \mathrm{ml}\end{array}$ \\
\hline IV & $\begin{array}{l}\text { Línea media luxada }>5 \mathrm{~mm} \text { : Desplazamiento de línea media }>5 \mathrm{~mm} \\
\text { con cisternas comprimidas o ausentes, no presencia de lesiones hiper- } \\
\text { densas o mixtas }>25 \mathrm{ml}\end{array}$ \\
\hline V & $\begin{array}{l}\text { Lesión focal }>25 \mathrm{ml} \text { evacuada: Desplazamiento de línea media }>5 \\
\text { mm con cisternas comprimidas o ausentes y lesiones hiperdensas o } \\
\text { mixtas }>25 \mathrm{ml}\end{array}$ \\
\hline VI & Lesión focal no evacuada \\
\hline
\end{tabular}

Tabla 2.

Puntaje de Rotterdam para los pacientes con traumatismo encefálocraneo

A.

Cisternas:

- Normales

- Comprimidas

- Ausentes

B. Línea media:

- Ausente o $\leq 5 \mathrm{~mm}$

- $>5 \mathrm{~mm}$

C. Hematoma epidural (para casos con lesiones de masa

intracraneales mayores a $25 \mathrm{ml}$ ):

- Presente

- Ausente

D. Hemorragia subaracnoidea o intraventricular:

- Ausente

- Presente

0

1

son muy escasas aun las investigaciones en busca de establecer la utilidad de las mismas para la predicción del riesgo de empeoramiento de las lesiones traumáticas intracraneales detectadas en la TC inicial de los lesionados con TEC, hecho denominado por los autores de este trabajo como empeoramiento tomográfico significativo, aspecto fundamental en la decisión de cambios terapéuticos oportunos, lo que incluye la realización de tratamientos neuroquirúrgicos antes de la instalación de lesiones secundarias catastróficas del encéfalo.
El siguiente trabajo se realizó con el objetivo de evidenciar la utilidad de la escala de Rotterdam para la predicción del empeoramiento tomográfico significativo en una serie de pacientes con TEC.

\section{Material y Método}

\section{Diseño y muestra:}

Se practicó un estudio observacional, prospectivo a partir de una muestra de pacientes con TEC estudiada en el Hospital Universitario "Manuel Ascunce 
Domenéch", Camagüey, Cuba, desde el 1 de julio de 2011 al 31 de enero de 2012.

La muestra no probabilística se conformó de acuerdo a los siguientes criterios:

- Pacientes con TEC, mayores de 18 años, hospitalizados en la institución antes citada, con TC de cráneo realizada al ingreso.

- Pacientes en los que se realizó TC de cráneo de control durante el mismo periodo de hospitalización.

\section{Indicación de TC de cráneo:}

- En el servicio de urgencias a todo paciente con Glasgow menor a 15 puntos y en pacientes con Glasgow de 15 puntos con alguna de las siguientes características: TEC penetrante, cinemática de alta energía, signos neurológicos deficitarios (defecto motor, afasia, trastorno de nervios craneales, etcétera) o irritativos (signos meníngeos o convulsiones), síntomas neurológicos (cefalea o vómitos) intensos o persistentes, signos clínicos o radiológicos de fractura craneal, portadores de coagulopatías o tratamiento con anticoagulantes.

- Para la indicación de TC de cráneo de control se siguieron las siguientes pautas:

- Control puntual: en cualquier momento que se detectó disminución de al menos un punto en la escala de Glasgow, aparición de un nuevo signo focal o deterioro de los parámetros de la dinámica intracraneal cuando se monitoreó la PIC

- Control temprano (período de 8 a 12 horas de la TC inicial): en pacientes que no hubieran requerido tratamiento quirúrgico (incluyó la colocación de catéter intracraneal para monitoreo de la PIC), con TC de cráneo inicial realizada antes de las primeras seis horas del traumatismo (TC precoz). Pacientes con TC precoz que se mantenían sedados, con Glasgow menor a 8 puntos, con trastornos de la coagulación o tratados con anticoagulantes.

- Control habitual (a las 72 horas de la TC inicial): pacientes con Glasgow inicial menor de 14 puntos en los que no se registró una mejoría clínica según lo esperado, pacientes con escala de Marshall II o III en la TC inicial o como control posoperatorio.
No se indicó TC craneal de control en los lesionados que se mantuvieron con un Glasgow superior a 13 puntos, con TC de cráneo inicial tipo I de acuerdo a la clasificación de Marshall y que mostraron mejoría clínica según lo esperado.

Los estudios imagenológicos con TC de cráneo fueron evaluados por un equipo conformado por neurorradiólo- gos y neurocirujanos de la institución rectora.

\section{Variables:}

Para cada paciente estudiado se llenó un formulario de forma sistemática por los investigadores que incluyó una serie de variables que se muestran operacionalizadas, el valor asignado aumentó en relación a la mayor gravedad:

\begin{tabular}{|c|c|}
\hline Variables & Codificación \\
\hline Grupos de edades & $\begin{array}{l}15-30=1 \\
31-46=2 \\
47-61=3 \\
62-76=4 \\
\text { más de } 77=5\end{array}$ \\
\hline Sexo & $\begin{array}{l}\text { femenino }=1 \\
\text { masculino }=2\end{array}$ \\
\hline Antecedentes patológicos personales & $\begin{array}{l}\text { no }=0 \\
\text { enfermedades crónicas compensadas }=1 \\
\text { alcoholismo }=2\end{array}$ \\
\hline Mecanismo del traumatismo & $\begin{array}{l}\text { cerrado de baja velocidad }=1 \\
\text { cerrado de alta velocidad }=2\end{array}$ \\
\hline Atención prehospitalaria & $\begin{array}{l}\text { no } \text { necesaria }=0 \\
\text { adecuada }=1 \\
\text { no adecuada }=2\end{array}$ \\
\hline Síntomas al ingreso & $\begin{array}{l}\text { no }=0 \\
\text { cefalea y vértigos }=1 \\
\text { amnesia peritraumática = } 2 \\
\text { vómitos }=3 \\
\text { agitación }=4 \\
\text { convulsión }=5\end{array}$ \\
\hline Escala de Glasgow inicial & $\begin{array}{l}15-14=1 \\
13-9=2 \\
\text { menos de } 9 \text { puntos }=3\end{array}$ \\
\hline Signos al ingreso & $\begin{array}{l}\text { no }=0 \\
\text { epistaxis o equimosis }=1 \\
\text { asimetría de reflejos }=2 \\
\text { rigidez nucal }=3 \\
\text { defecto motor larvado }=4 \\
\text { defecto motor evidente o disfasia }=5 \\
\text { trastornos ventilatorios o hemodinámicos }=6\end{array}$ \\
\hline Tiempo de realización de TC inicial & $\begin{array}{l}\text { antes de } 2 \text { horas }=1 \\
2-6 \text { horas }=2 \\
7-12 \text { horas }=3 \\
13-24 \text { horas }=4 \\
\text { más de } 24 \text { horas }=5\end{array}$ \\
\hline Escala de Marshall al ingreso & $\begin{array}{l}\text { tipo I = } 1 \\
\text { tipo II = } 2 \\
\text { tipo III = } 3 \\
\text { tipo IV = } \\
\text { tipo V }=5\end{array}$ \\
\hline Puntaje de Rotterdam al ingreso & $\begin{array}{l}0 \text { puntos }=0 \\
1 \text { punto }=1 \\
2 \text { puntos }=2 \\
3 \text { puntos }=3 \\
4 \text { puntos }=4 \\
5 \text { puntos }=5\end{array}$ \\
\hline Momento de la TC craneal de control & $\begin{array}{l}\text { hasta } 8 \text { horas }=1 \\
8-12 \text { horas }=2 \\
13-24 \text { horas }=3 \\
25-72 \text { horas }=4 \\
\text { más de } 72 \text { horas }=5\end{array}$ \\
\hline Cambios con la TC craneal de control & $\begin{array}{l}\text { no cambios }=1 \\
\text { aumento de volumen no significativo }=2 \\
\text { aumento de volumen significativo }=3\end{array}$ \\
\hline
\end{tabular}




\section{Procesamiento estadístico:}

Se construyó una base de datos usando el paquete estadístico SPSS 18.0. Se usaron técnicas de estadística exploratoria (frecuencia absoluta y por ciento). Para las técnicas de estadística confirmatoria se usó un intervalo de confianza del 95\%, e incluyeron los estadígrafos regresión logística binomial y ANOVA. Se tomó como variable dependiente la detección de empeoramiento significativo con la TC de cráneo de control.

\section{Aspectos Éticos:}

El estudio fue aceptado por la comisión de ética de la institución hospitalaria rectora de la investigación. Los pacientes fueron atendidos según guías de manejo aceptadas a nivel mundial, aplicadas de manera consensuada por el servicio de neurocirugía. Los datos de los enfermos se mantuvieron en confidencialidad.

\section{Resultados}

La muestra quedó constituida por 84 pacientes. En 61 (72,6\%) de los mismos no se registraron progresiones de las lesiones en la TC de control que motivaran cambios terapéuticos. En 13 $(15,5 \%)$ el empeoramiento de las lesiones detectado en la TC de control motivó la adopción de tratamiento quirúrgicos inmediatos, antes de la ocurrencia de degradación clínica, en uno de estos pacientes la cirugía consistió solo en la instalación de captor de presión intracraneal, en el resto dicho tratamiento neuroquirúrgico comprendió la realización de craneotomías con fines evacuadores o descompresivos. En 10 $(11,9 \%)$ enfermos se llevaron a cabo cambios en el tratamiento farmacológico, fundamentalmente la aplicación de osmoterapia, apoyados en la información de la TC de cráneo de control. (Figura 1).

En el modelo probabilístico determinado por técnica de regresión logística binomial, se comprobó que la introducción del puntaje de Rotterdam es significativo desde el punto de vista estadístico, para el pronóstico del empeoramiento de las lesiones intracraneales con repercusión terapéutica, detectadas en la TC de cráneo. (Tabla 3).

Utilizando una técnica de ANOVA se relacionó el puntaje de Rotterdam con la ocurrencia de empeoramiento ima- genológico significativo en esta serie. Se comprobó que el mismo se presentó con valores de dicho puntaje igual o superior a 1 y de manera mucho más evidente en los pacientes con puntaje 3. (Figura 2).
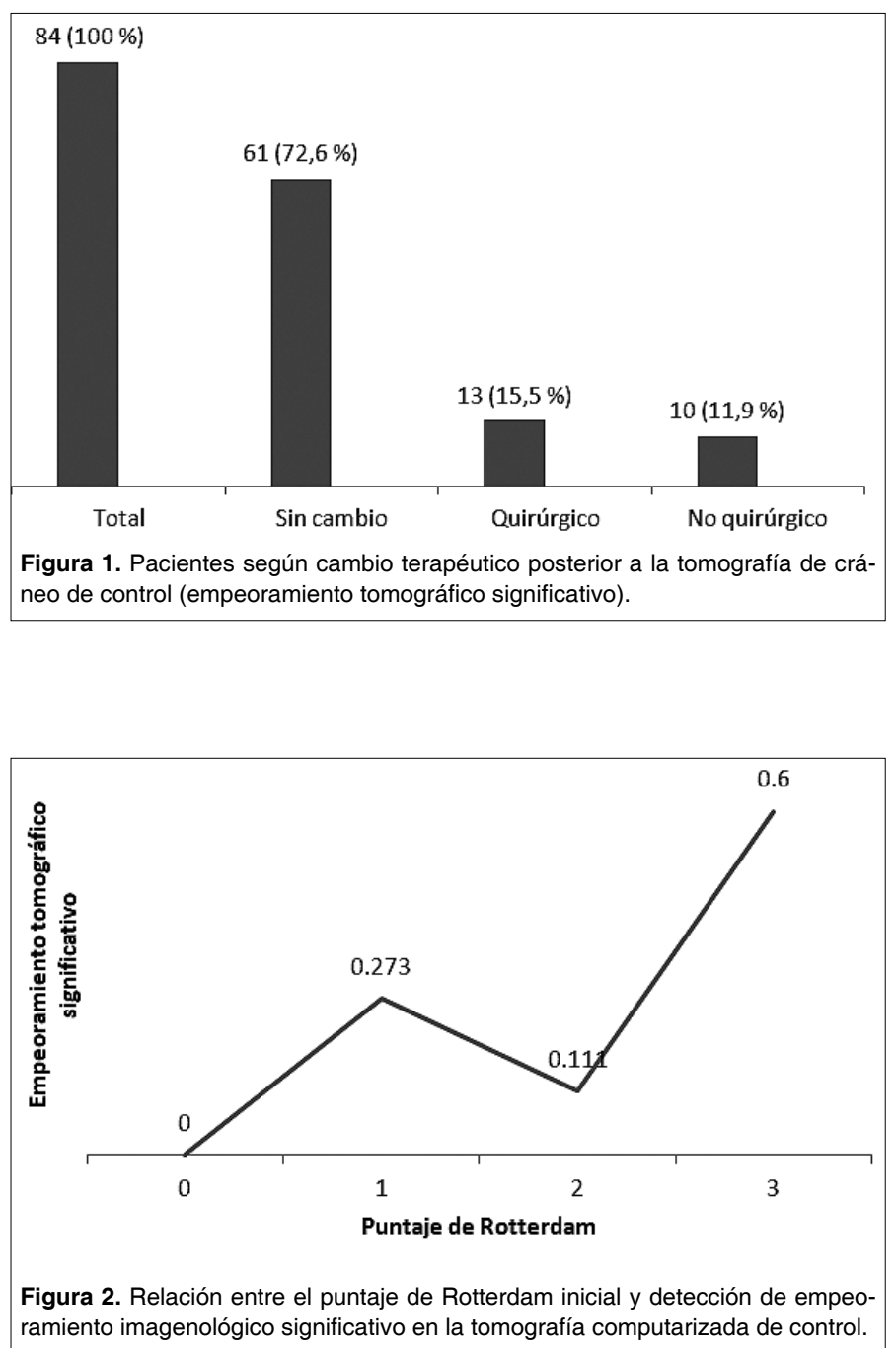

Tabla 3.

Resumen del modelo pronóstico del puntaje de Rotterdam para el empeoramiento significativo en la tomografía de cráneo de control

\begin{tabular}{|c|c|c|c|c|c|c|c|}
\hline Variable & \multirow{2}{*}{\multicolumn{2}{|c|}{ Observado }} & \multicolumn{5}{|c|}{ Pronosticado } \\
\hline \multirow{3}{*}{$\begin{array}{l}\text { Rotterdam } \\
\text { inicial }\end{array}$} & & & \multicolumn{2}{|c|}{$\begin{array}{l}\text { Cambio } \\
\text { tomográfico } \\
\text { significativo }\end{array}$} & $\begin{array}{l}\% \\
\text { correcto }\end{array}$ & $\begin{array}{l}\text { Valor } \\
\text { de } p\end{array}$ & I.C $95 \%$ \\
\hline & $\begin{array}{l}\text { Cambio } \\
\text { tomográfico } \\
\text { significativo }\end{array}$ & $\begin{array}{l}\text { No } \\
\text { Si }\end{array}$ & $\begin{array}{l}\mathrm{Si} \\
16 \\
3\end{array}$ & $\begin{array}{l}\text { No } \\
10 \\
4\end{array}$ & $\begin{array}{l}61,5 \\
57,1\end{array}$ & \multirow[t]{2}{*}{0,04} & \multirow[t]{2}{*}{$0,95-6,47$} \\
\hline & $\%$ global & & & & 60,6 & & \\
\hline
\end{tabular}




\section{Discusión}

Importancia del monitoreo del empeoramiento imagenológico significativo:

La introducción del neuromonitoreo en la atención de los pacientes con TEC en la década del 60 del pasado siglo marcó una nueva etapa de desarrollo, donde el principal objetivo se encamina a la adopción de médicas terapéuticas oportunas, antes de la ocurrencia de lesiones secundarias o al menos corregir las mismas antes de la instalación de lesiones encefálicas catastróficas.

A pesar de la multiplicidad de técnicas que incluyen determinaciones de la elastancia y complianza cerebrales, flujo sanguíneo, metabolismo, electrofisiológicas, etcétera; el monitoreo estructural del encéfalo mediante la TC de cráneo es una de las más empleadas a escala mundial. En este sentido es muy importante comprender que lo relevante para variar el pronóstico de los enfermos no es documentar el mero cambio en la imagen tomográfica, sino aquellos que implican un riesgo de empeoramiento clínico, hecho que los autores de este trabajo denominamos empeoramiento tomográfico significativo.

Por otro lado, la forma de realizar el control de los lesionados con TC de cráneo no está regida por estándares. Probablemente en muchos centros de neurotrauma esto se aplique según la opinión de uno o un grupo de neurocirujanos, al enfrentarse a la atención de cada caso en particular. Los autores de este trabajo han mostrado en otras publicaciones, un algoritmo basado en análisis estadísticos de causalidad, que ha resultado útil en el contexto local ${ }^{11}$.

En este trabajo se demuestra que con la aplicación del control con TC de cráneo de forma sistematizada, tal y como se explicó en el material y método, se logró la adopción de cambios terapéuticos oportunos, incluyendo la decisión de tratamientos neuroquirúrgicos en un número importante de enfermos, de manera independiente a la aplicación de cualquier otra técnica de neuromonitoreo, aspecto que en nuestra opinión no resta la importancia del neuromonitoreo multimodal.

Valor del puntaje de Rotterdam para el pronóstico de empeoramiento tomográfico significativo en pacientes con TEC:

A pesar de que la escala tomográfica de Marshall se ha mantenido como la más usada para el tamizaje patoanatómico de los pacientes con TEC, algunos aspectos negativos gravitan sobre la misma. En primer lugar no fue diseñada para el uso pronóstico y no representa una escala ordinal o sistema de gradación desde el punto de vista estadístico, además no toma en cuenta la presencia de hemorragia subaracnoidea ni intraventricular, no categoriza el grado de compresión de las cisternas basales, ni establece diferencias en cuanto a los tipos de lesiones intracraneales con efecto de masa ${ }^{2,13}$.

Por otro lado, el puntaje de Rotterdam fue diseñado con el objetivo de crear una escala ordinal, con la inclusión de la presencia o no de hemorragias subaracnoidea o intraventricular, diferenciando el hematoma extradural del resto de las lesiones intracraneales con efecto de masa y ofreciendo categorías diferentes según el grado de compromiso de las cisternas perimesencefálicas $^{12}$.

Varios estudios han demostrado la utilidad e inclusive la superioridad de esta escala, para la predicción de mortalidad y los malos resultados a corto y mediano plazo de los pacientes con TEC, después de la realización de craniectomía descompresiva e inclusive en pacientes pediátricos ${ }^{5,6,15}$. No obstante en la revisión sobre el tema llevada a cabo por los autores, no se encontró ninguna referencia que abordara la posible utilidad de la misma para realizar el pronóstico de la progresión de las lesiones intracraneales detectadas en la TC inicial, si bien Flint et al (citado por Fujimoto et al $)^{3}$ encontraron que la severidad del puntaje de Rotterdam en la TC inicial se asoció al riesgo de expansión de las contusiones encefálicas después de realizar craniectomía descompresiva.

En este estudio se encontró relación entre la severidad del puntaje de Rotterdam de la TC inicial y el riesgo de empeoramiento tomográfico significativo en la TC de control en los lesionados con TEC, hecho que consideramos muy relevante para la planificación más estrecha de los controles clínicos e imagenológicos en estos enfermos, de manera tal que puedan ser tomadas decisiones terapéuticas oportunas.

En esta investigación se detectó que dicho riesgo fue más evidente en los enfermos que alcanzaron puntaje de 3. Reza et al ${ }^{9}$, encontraron que los pa- cientes con puntaje de 4 mostraron mayor mortalidad a los 14 días después del TEC; vale aclarar que debido al diseño de dicho estudio, que incluyó la suma de 1 punto a cada categoría del Rotterdam para lograr su comparación con los 6 tipos de lesiones de la escala de Marshall, el valor de 4 es equivalente al de 3 de nuestra serie. En el ya citado trabajo de Fujimoto et al, se encontró mejor resultado en los pacientes tratados mediante craniectomía descompresiva con puntaje de Rotterdam inferior a 4.

\section{Escalas tomografías noveles:}

A pesar de las ya comentadas ventajas delaescaladeRotterdamcon respectoal Marshall, existen una serie de aspectos de potencial relevancia que no están incluidos en ninguna de las mismas; por ejemplo la distribución y volumen de la hemorragia subaracnoidea, las diferencias entre cada topografía de las lesiones intracraneales con efecto de masa, la existencia de signos de lesión axonal traumática, infartos del encéfalo, hemorragia del tallo cerebral y del llamado "cerebro negro" en la TC de cráneo.

En este sentido en los últimos años han sido publicadas otras escalas, más granuladas en relación a las variables imagenológicas con respecto a sus predecesoras, que persiguen mejorar estas falencias. La escala de Estocolmo se generó en 2010, única hasta el momento surgida de un estudio prospectivo; en la misma se usa la luxación de la línea media como una variable continua, se ofrece un puntaje a la hemorragia subaracnoidea y considera los signos de lesión axonal traumáti$\mathrm{Ca}^{10}$. (Tabla 4).

En el año 2014, se publica la escala de Helsinki. La misma incluye información recogida en las de Marshall y Rotterdam, pero brinda mayor relevancia al tipo específico de lesión intracraneal traumáticas y evalúa el estado de la cisterna supraselar, ${ }^{8,14}$. (Tabla 5).

Los estudios encaminados a la validación externa de dichas escalas son aun escasos, sin embargo, podrían resultar aportes en la estimación del pronóstico en cuanto a resultados y para la estimación del empeoramiento tomográfico significativo de los pacientes con TEC.

\section{Limitaciones del estudio:}

Hay que tener en cuenta que se ha 
Tabla 4.

Puntaje de Estocolmo

\begin{tabular}{l|c} 
Variables & Puntaje \\
\hline *HSAt: & \\
- Convexidad: & \\
$1-5 \mathrm{~mm}$ & 1 \\
$>5 \mathrm{~mm}$ & 2 \\
- Basal: & 1 \\
$1-5 \mathrm{~mm}$ & 2 \\
> $5 \mathrm{~mm}$ & \\
*HIV presente &
\end{tabular}

Ecuación: Luxación de línea media $(\mathrm{mm}) / 10+$ puntaje HSAt/2

-1 si hematoma epidural

+1 si lesión axonal traumática

+1 si hematoma subdural

+2 si hematoma subdural bilateral

Leyenda: HSAt: hemorragia subaracnoidea traumática, HIV: hemorragia intraventricular.

Tabla 5.

Puntaje de Helsinki

\begin{tabular}{l|c|}
\hline Variable & Puntaje \\
\hline *Tipo de hematoma: & \\
- Epidural & -3 \\
- Subdural & 2 \\
- Intraparenquimatoso & 2 \\
*Volumen de hematoma > 25 ml & 2 \\
*HIV presente & 3 \\
*Cisterna supraselar: & 0 \\
- Normal & 1 \\
- Comprimida & 5 \\
- Ausente & \\
\hline Leyenda: HIV: hemorragia intraventricular. &
\end{tabular}

estudiado una serie pequeña de pacientes, no se aquilata la relevancia de cada subcomponente de la escala de Rotterdam, ni se evalúa la relación de los mismos según los diferentes grados de severidad clínicos (leve, moderado - grave) de los pacientes con TEC. Dichas observaciones, junto con otras potencialmente válidas, evidencian la necesidad de generar investigaciones más abarcadoras en este sentido.

\section{Conclusiones}

En la estimación del pronóstico de los pacientes con TEC no solo es relevante lo relacionado con la mortalidad o los malos resultados, sino también con el riesgo de amplificación relevante de las lesiones intracraneales detectadas en la TC de cráneo inicial, lo cual brinda la posibilidad de generar cambios terapéuticos más oportunos. En este sentido constituye un factor de riesgo la elevación del puntaje de Rotterdam en la TC inicial.

\section{Recibido: 19 de diciembre de 2017} Aceptado: 02 de febrero de 2018

\section{Referencias}

1. Charry DJ, Falla DJ, Ochoa DJ, Pinzón AM, Tejeda HJ, Henríquez JM, et al. External Validation of the Rotterdam Computed Tomography Score in the Prediction of Mortality in Severe Traumatic Brain Injury. J Neurosci Rural Pract. 2017; 8 (Suppl 1): S23-S26. Doi: 10.4103/ jnrp.jnrp 43416.

2. Deepika A, Prabhuraj RA, Sarikia A, Shukla D. Comparison of predictability of Marshall and Rotterdam CT scan scoring system in determining early mortality after traumatic brain injury. Acta Neurochir. 2015; 157: 2033-38. Doi: 10.1007/s00701-015-2575-5.

3. Fujimoto K, Miura M, Otsuka T, Kuratsu J. Sequential changes in Rotterdam CT scores related to outcomes for patients with traumatic brain injury who undergo decompressive craniectomy. J Neurosurg. 2016; 124: 1640-45. Doi: 10.3171/2015.4.JNS142760.

4. Kasprowicz M, Burzynska M, Malcer T, Kübler A. A comparison of the Full Outline of Unresponsiveness (FOUR) and Glasgow Coma Scale (GCS) in predictive modelling in traumatic brain injury. British Journal of Neurosurgery. 2016; 30(2): 211-20. Doi: 10.3109/026886972016.1161173.

5. Liesemer K, Riva-Cambrin J, Statler BK, Bratton LS, Tran H, Metzger RR, et al. Use of Rotterdam CT Scores for Mortality Risk Stratification in Children with Traumatic Brain Injury. Pediatr Crit Care Med. 2014; 15 (6): 554-62. Doi: 10.1097/PCC.0000000000000150.

6. Mata-Mbemba D, Mugikura Sh, Nakagawa A, Murata T, Ishii K, Li L, et al. Early CT Findings to Predict Early Death in Patients with Traumatic Brain Injury: Marshall and Rotterdam CT Scoring System Compared i the Major Academic Tertiary Care Hospital in Northeastern Japan. Acad Radiol. 2014; 21(5): 605-11. Doi: 10.1016/j.acra.2014.01.017.

7. Munakomi S, Bhattarai B, Srinivas B, Cheriau I. Role of computed tomography scores and finfings to predictearly death in patients with traumatic brain injury: A reappraisal in a major tertiary care hospital in Nepal. Surg Neurol Int. 2016; 7: 23-37. Doi: 10.4103/21527806.177125 . 
8. Raj R. Siironem J, Skrifvars BM, Hernesniemi J, Kivisari R. Predicting Outcome in Traumatic Brain Injury: Development of a Novel Computerized Tomography Score. Neurosurg. 2014; 75: 632-47. Doi: 10.1227/NEU0000000000000533.

9. Reza TH, Fakharian E, Mousavi N, Abedzadeh KM, Akbari H, Zoghi S. The Rotterdam Scoring System Can Be as an Independent Factor for Predicting Traumatic Brain Injury Outcomes. World Neurosurg. 2016; 87: 195-199. Doi: 10.1016/j.wneu.2015.11.055.

10. Thelin PE, Nelson WD, Vehuilämen J, Nyström KR, Siironem J, Svensson M, et al. Evaluation of novel computerized tomographyscoring systems in human traumatic brain injury: An observational, multicenter study. PLoS Med. 2017; 14(8): e1002368. Doi: 10.1371/journal. pmed.1002368.

11. Varela HA, Martínez TC, Muñoz GR, Torres AR, Orellana CF, Lamus AL, et al. Algoritmo para la tomografía secuencial de cráneo en pacientes con traumatismo encéfalocraneano. Revista Chilena de Neurocirugía. 2016; 42(1): 24-30.

12. Waquas M, Shahzad SM, Faaiz ES, Qadeer M, Kamran BS, Patoli I, et al. Predicting outcomes of decompressive craniectomy: use of Rotterdam Computed Tomography Classification and Marshall Classification. British Journal of Neurosurgery. 2016; 30(2): 258-63. Doi: 10.3109/02688697.2016.1139047.

13. Waquas M, Kamran BS, Shahzad SM, Anwar S. Radiological prognostication in patients with head trauma requiring decompressive cranienctomy: Analysis of optic nerve sheath diameter and Rotterdam CT Scoring System. Journal of Neuroradiology. 2016; 43: 25-30. Doi: 10.1016/j.neurorad.2015.07.003.

14. Yao S, Song J, Li S, Cao C, Fang L, Wang C, et al. Helsinki Computed Tomography Scaning System Can Independently Predict LongTerm Outcome in Traumatic Brain Injury. World Neurosurg. 2017; 101: 528-33.Doi: 10.1016/j.wneu.2017.02.072.

15. Yu-Hua H, Yu-Han D, Tao-Chen L, Wu-Fu C. Rotterdam Computed Tomography Score as a Prognosticator in Head-Injury Patients Undergoing Decompresive Craniectomy. Neurosurg. 2012; 71: 80-85. Doi: 10.1227/NEU.0b013e3182517aaa1.

Conflicto de intereses: Sin conflicto de intereses.

\section{Correspondencia a:}

Dr. Ariel Varela Hernandez

varelahernandezariel@gmail.com 